\title{
Kinetic Analysis of Dynamic "C-Acetate PET/CT Imaging as a Potential Method for Differentiation of Hepatocellular Carcinoma and Benign Liver Lesions
}

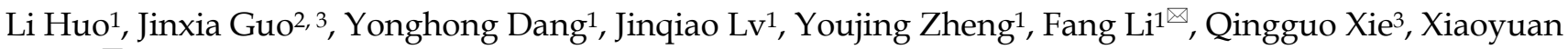 \\ Chen $^{2} \bowtie$ \\ 1. Department of Nuclear Medicine, Peking Union Medical College Hospital \\ 2. Laboratory of Molecular Imaging and Nanomedicine (LOMIN), National Institute of Biomedical Imaging and Bioengineering, National \\ Institutes of Health (NIBIB), Bethesda, Maryland, USA \\ 3. Department of Biomedical Engineering, and Wuhan National Laboratory for Optoelectronics(WNLO), Huazhong University of Science \\ and Technology, Wuhan, Hubei, China
}

$\triangle$ Corresponding authors: Fang Li, M.D., 1 Shuaifuyuan, Dongcheng District, Beijing, China, 100730. Email: lifang@pumch.cn. Xiaoyuan Chen, Ph.D., 35A Convent Dr, GD937, Bethesda, MD 20892-2281. Email: shawn.chen@nih.gov

(c) Ivyspring International Publisher. This is an open-access article distributed under the terms of the Creative Commons License (http://creativecommons.org/ licenses/by-nc-nd/3.0/). Reproduction is permitted for personal, noncommercial use, provided that the article is in whole, unmodified, and properly cited.

Received: 2014.10.08; Accepted: 2014.12.27; Published: 2015.01.21

\begin{abstract}
Objective: The kinetic analysis of " $\mathrm{C}$-acetate PET provides more information than routine one time-point static imaging. This study aims to investigate the potential of dynamic " $\mathrm{C}$-acetate hepatic PET imaging to improve the diagnosis of hepatocellular carcinoma (HCC) and benign liver lesions by using compartmental kinetic modeling and discriminant analysis.

Methods: Twenty-two patients were enrolled in this study, 6 cases were with well-differentiated $\mathrm{HCCs}, 7$ with poorly-differentiated HCCs and 9 with benign pathologies. Following the CT scan, all patients underwent ${ }^{~ " C} \mathrm{C}$-acetate dynamic PET imaging. A three-compartment irreversible dual-input model was applied to the lesion time activity curves (TACs) to estimate the kinetic rate constants $K_{1}-k_{3}$, vascular fraction (VB) and the coefficient $\alpha$ representing the relative hepatic artery (HA) contribution to the hepatic blood supply on lesions and non-lesion liver tissue. The parameter $\mathrm{Ki}\left(=\mathrm{K}_{1} \times \mathrm{k}_{3} /\left(\mathrm{k}_{2}+\mathrm{k}_{3}\right)\right)$ was calculated to evaluate the local hepatic metabolic rate of acetate (LHMAct). The lesions were further classified by discriminant analysis with all the above parameters.

Results: $\mathrm{K}_{1}$ and lesion to non-lesion standardized uptake value (SUV) ratio (T/L) were found to be the parameters best characterizing the differences among well-differentiated HCC, poorly-differentiated HCC and benign lesions in stepwise discriminant analysis. With discriminant functions consisting of these two parameters, the accuracy of lesion prediction was $87.5 \%$ for well-differentiated HCC, $50 \%$ for poorly-differentiated HCC and $66.7 \%$ for benign lesions. The classification was much better than that with SUV and T/L, where the corresponding classification accuracy of the three kinds of lesions was $57.1 \%, 33.3 \%$ and $44.4 \%$.

Conclusion: " $\mathrm{C}$-acetate kinetic parameter $\mathrm{K}_{1}$ could improve the identification of $\mathrm{HCC}$ from benign lesions in combination with $T / L$ in discriminant analysis. The discriminant analysis using static and kinetic parameters appears to be a very helpful method for clinical liver masses diagnosis and staging.
\end{abstract}

Key words: ${ }^{11} \mathrm{C}$-Acetate, dynamic PET; hepatocellular carcinoma; kinetic modeling; discriminant analysis 


\section{INTRODUCTION}

Reliable staging of liver lesions is the prerequisite to establish the optimal treatment and determine the prognosis [1-3]. Both ${ }^{18} \mathrm{~F}-\mathrm{FDG}$ and ${ }^{11} \mathrm{C}$-acetate PET imaging have demonstrated the ability to detect and stage hepatocellular carcinoma (HCC) [4-6]. Due to the high expression of the glucose-6-phosphatase enzyme that prevents intracellular accumulation of ${ }^{18} \mathrm{~F}-\mathrm{FDG}$, about $40-50 \%$ of $\mathrm{HCC}$ are not sensitive to ${ }^{18} \mathrm{~F}-\mathrm{FDG}$ PET [4]. In comparison, ${ }^{11} \mathrm{C}$-acetate, which is believed to mainly participate in fatty acid synthesis in the liver, has been considered as a more promising tracer than ${ }^{18} \mathrm{~F}-\mathrm{FDG}$ in terms of detection sensitivity and specificity, especially for well-differentiated HCC $[4,6,7]$.

However, the benign hepatic lesions, such as the focal nodular hyperplasia (FNH), hepatic adenoma and inflammation, with a much higher occurrence rate than the malignant tumors [8], also accumulate ${ }^{11} \mathrm{C}$-acetate. There have been reports that ${ }^{11} \mathrm{C}$-acetate PET is poor in differentiating HCC from benign lesions [6, 9, 10]. Dominique et al. [9] and Ho et al. [6] suggested in their studies that dynamic PET with kinetic modeling might improve the understanding of these lesions for separating benign hepatic tumors from HCC. The fast kinetics of acetate is an advantage of ${ }^{11} \mathrm{C}$-acetate over ${ }^{18} \mathrm{~F}$-FDG, which makes the duration of dynamic imaging short and can be endured by the patients. Chen et al. applied an irreversible three-compartment model with dual input function from hepatic artery (HA) and hepatic portal vein (PV) to study the ${ }^{11} \mathrm{C}$-acetate kinetics in the liver [11]. In contrast to the routine one time-point static imaging, quantitative tracer transport rate $\mathrm{K}_{1}-\mathrm{k}_{3}$, the local hepatic metabolic rate constant of ${ }^{11} \mathrm{C}$-acetate (LHMRAct) and the fraction of HA blood supply from the dynamic modeling were calculated to characterize HCC and benign liver lesions in a more comprehensive manner.

There are limited studies investigating systematically acetate kinetics in liver HCC and benign lesions. In this study, we performed ${ }^{11} \mathrm{C}$-acetate dynamic PET on patients with liver lesions and conducted the kinetic modeling. The purpose is to explore the variance of quantitative parameters between HCC and benign lesions and investigate whether the differentiation can be improved when kinetic parameters is introduced in discriminant analysis [12-14].

\section{MATERIALS AND METHODS}

\section{Patients}

The study was approved by the Ethics Committee of Peking Union Medical College Hospital. The informed consent was obtained from all the patients prior to the study. Twenty-two patients suffered from liver lesion (16 males and 6 females; age range, 40-79 $y$; mean $\pm S D, 55.7 \pm 11.9$ y) were enrolled in the study. All the lesions including HCC and benign lesions were confirmed by surgical resection and pathological examination within two weeks after ${ }^{11} \mathrm{C}$-acetate PET/CT, and graded according to the well-known Edmondson-Steiner criteria $[15,16]$.

\section{C-Acetate Synthesis/Preparation}

${ }^{11} \mathrm{C}$-acetate was synthesized by transferring the ${ }^{11} \mathrm{C}-\mathrm{CO}_{2}$ generated by Siemens RDS111 cyclotron into a fully automated radiochemistry module based on the procedure described by Mitterhauser et al. [17]. The chemical and radiochemical purities were both greater than $98 \%$. The product was proved to be sterile by $24 \mathrm{~h}$ bacterial culture.

\section{PET/CT and Enhanced CT Imaging}

PET/CT scans were conducted on the Siemens Biography 64 True Point PET/CT scanner (Siemens Medical Solution, Inc.) for all patients. CT transmission scans were acquired first for anatomy observation and providing the data for PET attenuation and scatter correction. Subsequently, the patient was scanned over the left ventricle and the whole liver. About $7 \mathrm{MBq} / \mathrm{kg}(\sim 0.19 \mathrm{mCi} / \mathrm{kg}){ }^{11} \mathrm{C}$-acetate was administered intravenously to each patient and the dynamic PET data were acquired simultaneously. Each PET scan lasted $10 \mathrm{~min}$ and the list mode data were reconstructed with 2D-OSEM [11, 18, 19]. The reconstruction frames consisted of $12 \times 10 \mathrm{~s}, 9 \times 20 \mathrm{~s}$, $1 \times 60 \mathrm{~s}$, and $2 \times 120 \mathrm{~s}$. As a reference, the enhanced CT imaging was also done before the PET/CT scan and used for the blood supply observation in liver masses.

\section{Image Analysis}

Sequential CT and PET images were accurately registered on Inveon Research Workplace (IRW) 3.0 software (Siemens Preclinical Solution) for further quantification. The regions of interest (ROIs) were drawn over lesions and non-lesion regions slice-by-slice on PET/CT images of the last frame with the help of experienced clinicians. Final volume of interest (VOI) was created by combining all the ROIs $(\mathrm{n}=5 \sim 8)$ for each patient. The average decay-corrected activities in the VOIs were converted to the standardized uptake value (SUV) using the following calculation: activity concentration $(\mathrm{kBq} / \mathrm{ml}) /$ (injection dose $(\mathrm{MBq}) /$ body weight $(\mathrm{kg})$ ). To generate the lesion time-activity curves (TACs) for dynamic analysis, the VOIs on the last frame were superimposed to all the other frames in dynamic image sequences for each patient and the SUV at each 
time point was calculated. The partial-volume effect was ignored in the study since all the lesions had sizes $(\geq 1 \mathrm{~cm})$ that were more than three times the PET spatial resolution $(\sim 2 \mathrm{~mm})[19,20]$.

Since liver has blood supply from both hepatic artery (HA, with oxygenated blood) and portal vein (PV, with nutrient rich blood), both the HA and PV TACs were used as the input function in ${ }^{11} \mathrm{C}$-acetate kinetic modeling. HA TAC was obtained from the abdominal aorta near the liver [11] through a VOI defined on the image frame where the peak tracer accumulation was observed. The VOIs for PV were defined over the PV based on the aligned CT images.

\section{C-Acetate Kinetics Modeling and Parameters Estimation in Liver}

${ }^{11} \mathrm{C}$-acetate can be quickly taken up by the cell and converted into the ${ }^{11} \mathrm{C}$-acetyl-CoA by acetate kinase. Then ${ }^{11} \mathrm{C}$-acetyl-CoA is converted to fatty acid or cholesterol, and participate in the formation of phosphatidylcholine membrane, or be metabolized to $\mathrm{CO}_{2}$ via the Kreb's cycle [21, 22]. Although the metabolism routes are not well understood, the former one is considered to be predominant in the liver and tumor cells [22-24]. Regarding the high ${ }^{11} \mathrm{C}$-acetate accumulation in lesions and dual blood supply from HA and $P V$ in the liver tissue, an irreversible three-compartment model with dual input has been confirmed previously to be appropriate for ${ }^{11} \mathrm{C}$-acetate kinetics in tumor and non-tumor liver tissue $[18,22]$.

The model can be described by the following equations:

$$
\begin{gathered}
\frac{d C_{f}(t)}{d t}=K_{1} \cdot C_{p}(t)-\left(k_{2}+k_{3}\right) \cdot C_{f}(t) \\
\frac{d c_{m}(t)}{d t}=k_{3} \cdot C_{f}(t) \\
C_{t}(t)=C_{f}(t)+C_{m}(t)+V_{b} \cdot C_{p}(t) \\
C_{p}(t)=\alpha C_{H A}(t)+(1-\alpha) C_{P V}(t)
\end{gathered}
$$

Where $C_{p}$ is the tracer concentration in the first compartment and used as the input function which is contributed from both hepatic artery $\left(\mathrm{C}_{\mathrm{HA}}\right)$ and hepatic portal vein $\left(\mathrm{C}_{\mathrm{PV}}\right)$ as shown in Eq. 4 where $\alpha$ is the contribution ratio. $C_{f}$ and $C_{m}$ represent the tracer concentrations in the interstitial and the intracellular spaces - the second and third compartment, respectively. $C_{t}$ is the tracer concentration in the entire tissue VOI including the $C_{f}, C_{m}$ and part of $C_{p}$ in Eq. 3. $V_{b}$ in Eq. 3 is the fractional blood volume.

Kinetic rate constant $K_{1}$ refers to the tracer perfusion rate from plasma to the tissue and $k_{2}$ means the tracer clearance rate back to the plasma. $\mathrm{k}_{3}$ is associated with the rate of ${ }^{11} \mathrm{C}$-acetate metabolism. $\mathrm{K}_{1}-\mathrm{k}_{3}, \alpha$ and $\mathrm{V}_{\mathrm{b}}$ are derived by fitting tumor or non-tumor tissue TACs with un-weighted least-squares optimization method according to Eq. 1- Eq. 4. An iterative method was used to adjust the estimated parameters to achieve the minimum least squares difference between the measured TACs and estimated TACs. The Akaike Information Criteria (AIC) was used to assess the fitting $[18,25]$. In addition, the combined parameter $\mathrm{K}_{1}\left(=\mathrm{K}_{1} \times \mathrm{k}_{3} /\left(\mathrm{k}_{2}+\mathrm{k}_{3}\right)\right)$, was also calculated to estimate the local hepatic metabolic rate of ${ }^{11} \mathrm{C}$-acetate (LHMRAct) [11, 18].

\section{Statistical Analysis}

Unpaired Student $t$-test was applied to all the static and dynamic parameters that potentially characterize the tumor or non-tumor tissues. The parameters include: (a) SUV; (b) SUV tumor to non-tumor liver tissue ratio (T/L); (c) $\mathrm{K}_{1}$; (d) $\mathrm{k}_{2}$; (e) $\mathrm{k}_{3}$; (f) $\alpha$; (g) LHMRAct $\left(\mathrm{K}_{1} \times \mathrm{k}_{3} /\left(\mathrm{k}_{2}+\mathrm{k}_{3}\right)\right)$. P value less than 0.05 was considered statistically significant.

\section{Discriminant Analysis}

Discriminant analysis with stepwise method and Fisher criteria [26-28] was conducted with the R statistical software (www.r-project.org). To identify the appropriate parameters that can best differentiate between the HCC and benign lesions, the Wilks' Lambda test was used to examine the mean values in each group. The parameters entering and removing from the analysis were determined with the typical $\mathrm{F}$ probability value of $<0.05$ and $>0.1$, respectively. Finally, the linear discriminant functions were constructed by the Fisher criteria with the classification variables to classify the lesions. The classification variables were imported into the above discriminant functions to calculate the discriminant score. The lesion was classified into the group whose discriminant function has the highest score. The classification results were further validated with the leave-one-out cross-validation.

\section{RESULTS}

\section{Patients}

By surgery and pathological analysis, 7 patients were proved to have well-differentiated HCC, 6 with poorly-differentiated HCC. Benign lesions were found in the remaining 9 patients, (3 focal nodular hyperplasia $(\mathrm{FNH}), 1$ hemangioma, 2 inflammation lesions, 1 lymphoid hyperplasia, 1 hepatic adenoma and 1 angiomyolipoma). In enhanced CT imaging, 4 well-differentiated HCC and 2 poorly-differentiated HCC lesions were regarded as benign lesions and all 10 benign lesions were diagnosed as well-differentiated HCC lesions due to their enhanced 
HA blood supply. An overview of the patients' information is summarized in Table 1.

Table 1. The characteristics of all patients

\begin{tabular}{|c|c|c|c|c|c|c|}
\hline Group & $\begin{array}{l}\text { Patient } \\
\text { No. }\end{array}$ & $\begin{array}{l}\text { Sex } \\
\text { (F/M) }\end{array}$ & $\begin{array}{l}\text { Age } \\
\text { (year) }\end{array}$ & $\begin{array}{l}\text { Tumor } \\
\text { size }(\mathrm{cm})\end{array}$ & $\begin{array}{l}\text { Cirrhosis } \\
\text { (yes/no) }\end{array}$ & Final diagnosis* \\
\hline I & 7 & $2 / 5$ & $57.4 \pm 13.9$ & $3.04 \pm 1.9$ & $7 / 0$ & Well-HCC \\
\hline II & 6 & $0 / 6$ & $61 \pm 13.6$ & $1.55 \pm 0.60$ & $4 / 2$ & Poor-HCC \\
\hline III & 9 & $4 / 5$ & $52 \pm 8.9$ & $1.93 \pm 1.16$ & $0 / 9$ & Benign tumor \\
\hline
\end{tabular}

\section{PET Images, SUV and Time Activity Curves}

The transaxial ${ }^{11} \mathrm{C}$-acetate PET/CT co-registered images at early and late time points for one patient with well-differentiated HCC were shown in Figure 1A where aorta, hepatic portal vein and the HCC lesion can be clearly distinguished. The average SUV in the different groups of lesions and non-lesion liver tissue and the lesion to non-lesion liver tissue SUV ratio (T/L) were shown in Supplementary Material: Figure S1. Significant differences $(\mathrm{P}<0.01)$ were found in average SUVs between benign lesions and non-lesion liver tissues, and between well-differentiated HCC and non-lesion liver tissues, as well as in the $\mathrm{T} / \mathrm{L}$ between the poorly-differentiated HCC and non-lesion liver tissue, and between well-differentiated HCC and non-lesion liver tissue. However, there were significant overlaps of SUV values in different groups, as well as overlapping T/L between HCC and benign lesions, making the lesion differentiation difficult.

The corresponding TACs of the patient in Figure 1A were plotted in Figure $1 \mathrm{~B}$. The TAC of PV showed slight delay in reaching maximum uptake as compared to HA [11, 29]. The TACs of aorta and portal vein had almost the same C-11 activity uptake values beyond 3 min post-injection, and a relative stable T/L was observed. For all the patients, the variance of $\mathrm{T} / \mathrm{L}$ was less than $15 \%$ during this 'stable period' $(3 \sim 10$ $\min )$.

\section{Kinetic Parameters}

As shown in Supplementary Material: Figure S2, kinetic parameters $K_{1}-k_{3}$ and $\alpha$ in different types of lesions and non-lesion liver tissues were compared. There was a significant difference between: (1) well-differentiated HCC $\mathrm{K}_{1}$ and benign lesion $\mathrm{K}_{1}(\mathrm{P}<$ $0.05)$; (2) poorly-differentiated $\mathrm{HCC} \mathrm{K}_{1}$ and benign lesion $\mathrm{K}_{1}(\mathrm{P}<0.05)$; (3) well-differentiated $\mathrm{HCC} \mathrm{k}_{3}$ and non-lesion liver tissue $\mathrm{k}_{3}(\mathrm{P}<0.01)$; well-differentiated HCC $\alpha$ and non-lesion liver tissue $\alpha(\mathrm{P}<0.01)$; (5) poorly-differentiated HCC $\alpha$ and non-lesion liver tissue $\alpha(\mathrm{P}<0.01)$; and (6) benign lesion $\alpha$ and non-lesion liver tissue $\alpha(\mathrm{P}<0.05)$.

Moreover, statistical analysis was also conducted on the LHMRAct $\mathrm{K}_{\mathrm{i}}$ (Supplementary Material: Figure S3). Significant difference was only found between well-differentiated $\mathrm{HCC}_{\mathrm{i}}$ and non-lesion liver tissue $\mathrm{K}_{\mathrm{i}}$.

\section{Classification by Discriminant Analysis}

With all the parameters derived from static and dynamic scans, Wilks' Lambda test was performed step by step as shown in Table 2 until there was no parameter with significant $F$ value. $K_{1}$ and $T / L$ were finally identified as the classification variables with the minimal $F$ value and Wilks' Lambda value. The linear Fisher discriminant function for well-differentiated HCC, poorly-differentiated HCC and benign lesions were defined as follows:

$Y_{\text {well }-H C C}=11.674 \cdot\left(\frac{\text { lesion }}{\text { liver }}\right)_{S U V}+5.481 \cdot K_{1}-14.916$

$Y_{\text {poor }-H C C}=7.790 \cdot\left(\frac{\text { lesion }}{\text { liver }}\right)_{S U V}+4.715 \cdot K_{1}-8.358$

$$
Y_{\text {benign }}=9.972 \cdot\left(\frac{\text { lesion }}{\text { liver }}\right)_{\text {SUV }}+7.746 \cdot K_{1}-15.120
$$

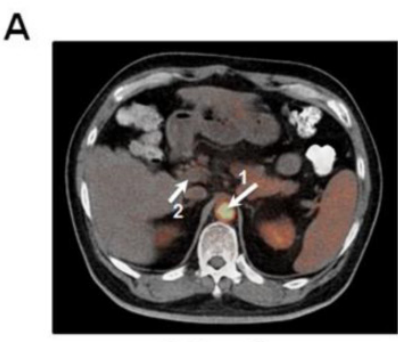

$0.5 \mathrm{~min}$

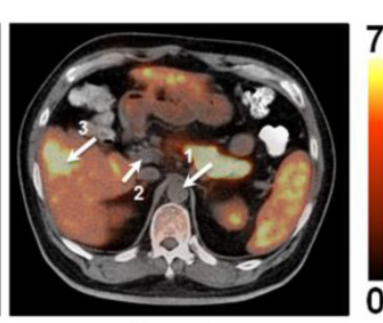

$8 \mathrm{~min}$
B

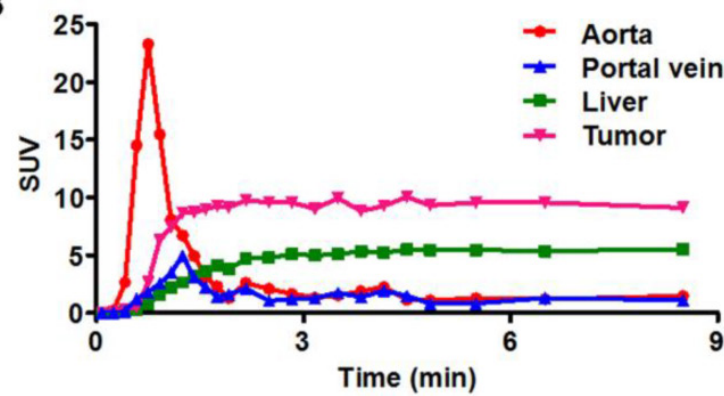

Figure 1. (A) The co-registered transaxial PET/CT images at $0.5 \mathrm{~min}$ and $8 \mathrm{~min}$ after injection of " $\mathrm{C}$-acetate into the patient with HCC. Arrows display the ROls for aorta (arrow 1), hepatic portal vein (arrow 2) and HCC (arrow 3). (B) The corresponding time activity curves (TACs) for ROls defined over aorta, hepatic portal vein, liver and the HCC. Tumor uptake in each time point is represented by standardized uptake value (SUV). 
The classification results for all the lesions were presented in Table 3. All but one patients with well-differentiated HCC were diagnosed correctly (1 patient was mis-diagnosed as benign lesion). The accuracy for poorly-differentiated HCC and benign lesions were $50 \%$ and $66.7 \%$, respectively. With leave-one-out cross-validation (Table 4), the classification accuracy of benign lesions was decreased to $55.6 \%$. Details of classification for each patient were displayed in Supplementary Material: Table S1.

To demonstrate the improvement of the classification with the kinetic parameters, we also conducted the discriminant analysis of the parameters from static imaging: SUV and the T/L. A worse classification was found with accuracy of $57.1 \%$ for well-differentiated HCC, $33.3 \%$ for poorly-differentiated HCC and $44.4 \%$ for benign lesions (Table 5).

Table 2. The Wilks' Lambda test

\begin{tabular}{llll}
\hline Steps & Parameters & F & Wilks Lambda \\
\hline 0 & $\mathrm{~K}_{1}$ & 0.017 & 0.649 \\
& $\mathrm{k}_{2}$ & 0.221 & 0.853 \\
& $\mathrm{k}_{3}$ & 0.088 & 0.775 \\
& HMRAct & 0.209 & 0.848 \\
& $\alpha$ & 0.205 & 0.846 \\
& SUV & 0.138 & 0.812 \\
& (Lesion/Liver) $_{\text {suv }}$ & 0.034 & 0.701 \\
1 & $\mathrm{k}_{2}$ & 0.966 & 0.647 \\
& $\mathrm{k}_{3}$ & 0.091 & 0.498 \\
& HMRAct & 0.082 & 0.492 \\
& $\alpha$ & 0.263 & 0.560 \\
& SUV & 0.157 & 0.529 \\
& (Lesion/Liver) $)_{\text {suv }}$ & 0.030 & 0.439 \\
& $\mathrm{k}_{2}$ & 0.949 & 0.436 \\
& $\mathrm{k}_{3}$ & 0.172 & 0.357 \\
& HMRAct & 0.219 & 0.367 \\
& $\alpha$ & 0.570 & 0.411 \\
& SUV & 0.582 & 0.412 \\
\hline
\end{tabular}

Table 3. The classification results of $\mathrm{HCC}$ and benign lesions with lesion to non-lesion SUV ratio and $K_{1}$ in discriminant analysis

\begin{tabular}{llll}
\hline \multicolumn{4}{l}{ Classification results (Num. / probability) } \\
\hline Total & Well-HCC & Poor-HCC & Benign \\
Well-HCC (7) & $6(85.7 \%)$ & 0 & $1(14.3 \%)$ \\
Poor-HCC (6) & $1(16.7 \%)$ & $3(50.0 \%)$ & $2(33.3 \%)$ \\
Benign (9) & $1(11.1 \%)$ & $2(22.2 \%)$ & $6(66.7 \%)$ \\
\hline
\end{tabular}

*Well-HCC: Well-differentiated HCC; Poor-HCC: Poorly-differentiated HCC.

Table 4. The classification results of $\mathrm{HCC}$ and benign lesions with leave-one-out cross-validation in discriminant analysis

\begin{tabular}{llll}
\hline \multicolumn{4}{l}{ Classification results (Num./ probability) } \\
\hline Total & Well-HCC & Poor-HCC & Benign \\
Well-HCC (7) & $6(85.7 \%)$ & 0 & $1(14.3 \%)$ \\
Poor-HCC (6) & $1(16.7 \%)$ & $3(50.0 \%)$ & $2(33.3 \%)$ \\
Benign (9) & $2(22.2 \%)$ & $2(22.2 \%)$ & $5(55.6 \%)$ \\
\hline *Well-HCC: Well-differentiated HCC; Poor-HCC: Poorly-differentiated HCC
\end{tabular}

*Well-HCC: Well-differentiated HCC; Poor-HCC: Poorly-differentiated HCC.
Table 5. The classification results of HCC and benign lesions with SUV and lesion to non-lesion SUV ratio in discriminant analysis

\begin{tabular}{llll}
\hline \multicolumn{4}{l}{ Classification results (Num. / probability) } \\
\hline Total & Well-HCC & Poor-HCC & Benign \\
Well-HCC (7) & $4(57.1 \%)$ & 0 & $3(42.9 \%)$ \\
Poor-HCC (6) & $0(0.0 \%)$ & $2(33.3 \%)$ & $4(66.7 \%)$ \\
Benign (9) & $3(33.3 \%)$ & $2(22.2 \%)$ & $4(44.4 \%)$ \\
\hline
\end{tabular}

*Well-HCC: Well-differentiated HCC; Poor-HCC: Poorly-differentiated HCC.

\section{DISCUSSION}

Our study confirmed that the irreversible three-compartment model with dual input is appropriate for analyzing dynamic ${ }^{11} \mathrm{C}$-acetate PET in the liver. Both static and kinetic parameters were imported into the stepwise discriminant analysis and it was found that $K_{1}$ and $T / L$ are the best classification variables to differentiate different types of lesions in the liver.

As reported in the study by Ho et al. [4], we also found hypo-metabolic foci of ${ }^{11} \mathrm{C}$-acetate in well differentiated HCC in which the tumor cells are more histologically similar to the normal liver cells. When the lesion is transformed to poorly differentiated HCC, ${ }^{11} \mathrm{C}$-acetate uptake is decreased. There is thus significant difference in $\mathrm{T} / \mathrm{L}$ between these two types of lesions. For benign liver lesions, there is a large variation in the $T / L$ ratio. Hence, $T / L$ ratio can be a good indicator for HCC staging, but not for the identification of benign lesions.

$\mathrm{K}_{1}$ ( $\mathrm{ml}$ blood/ $\mathrm{ml}$ liver tissue/ $\mathrm{min}$ ) represents the tracer transport rate from blood to liver tissue. Kinetic analysis found that $K_{1}$ value decreased with increased blood supply from HA for any ROI. Because ${ }^{11} \mathrm{C}$-acetate accumulation in $\mathrm{HA}$ is earlier and much higher than in PV at the beginning of tracer injection [29], HCC lesions with enhanced HA blood supply had lower $K_{1}$ to reach the tracer concentration in the 'stable period' than benign lesions and non-lesion liver tissue. That the HCC with similar HA blood supply as the benign or non-lesion liver tissue yet had low $K_{1}$ may result from the relative low tracer uptake. In general, $\mathrm{K}_{1}$ is a good reflection of HA and PV blood supply ratio and the tracer uptake in the liver tissue, allowing us to easily differentiate the HCC from benign lesions.

The fact that $k_{2}$ and $k_{3}$ did not appear significantly in the discriminant analysis may imply that the dynamic course in the 'stable period' in ${ }^{11} \mathrm{C}$-acetate dynamic imaging doesn't play an important role in differentiating HCC from benign lesions. The HA blood supply ratio was not good either for differentiation, because some HCC lesions did not show enhanced HA blood supply while some benign lesions were on the contrary, as found in studies of hepatic 
hemodynamics or perfusion [30-34], which explains why the benign lesions have been frequently mis-diagnosed as HCC in enhanced CT imaging.

LHMRAct $K_{i}$ is a parameter regarded not to be affected by the tissue microenvironment, such as the heterogeneity of vascular permeability and variance of blood flow or perfusion, thus more sensitive than SUV to reflect the real ${ }^{11} \mathrm{C}$-acetate metabolism [35]. The significant difference of LHMRAct between well-differentiated HCC and non-lesion liver tissue further indicated the well-differentiated HCC lesions were ${ }^{11} \mathrm{C}$-acetate avid. The benign lesions presented significant higher value than non-lesion liver tissue in SUV but not in $\mathrm{K}_{\mathrm{i}}$. It can be inferred that the tracer concentration was mainly from the ${ }^{11} \mathrm{C}$-acetate retention in interstitial space contributed by the high perfusion $\left(\mathrm{K}_{1}\right)$ in benign lesions, not the high ${ }^{11} \mathrm{C}$-acetate metabolism. Given the large overlapping value and the insignificant difference among the lesion groups, $\mathrm{Ki}$ was also unable to differentiate them.

Although the combination of the static and kinetic parameters could well characterize the liver lesions, it's difficult to differentiate the lesions by simple statistical analysis. The discriminant analysis was then introduced to do the lesions classification. The results with $\mathrm{T} / \mathrm{L}$ and $\mathrm{K}_{1}$ outperform that with static parameters, and showed best sensitivity was obtained for well-differentiated HCC, then for benign lesions. Comparable classification accuracy in leave-one-out cross-validation demonstrated the robustness of the discriminant functions. Poorly-differentiated HCC was not well identified even when kinetics analysis was added. To avoid these unsatisfactory results, ${ }^{18} \mathrm{~F}-\mathrm{FDG}$ imaging should be used for poorly-differentiated HCC diagnosis. Among the benign lesions in our patients, two inflammation lesions were regarded as the poorly-differentiated HCC, while 3 FNH lesions were correctly classified in the benign group. There is a possibility that our method is more useful for FNH lesions than for inflammation lesions. The angioma and benign lymphoid hyperplasia were classified correctly. The angiomyolipoma lesion, as well as the hepatic adenoma lesion, were mis-classified as the well-differentiated HCC in the leave-one-out-cross-validation. The small number of samples of each type of benign lesions was a limitation of our study (lack of statistical power). Study with more HCC and benign lesions are required in future.

Cirrhosis is usually thought to cause increased HA blood supply that makes the diagnosis of liver lesions more difficult $[4,36]$. In this study, 11 patients with HCC were found to have cirrhosis in liver tissue, but the SUV and $K_{1}$ in their non-lesion liver tissue ROI didn't show significant differences from normal liver tissue in other objects. Thus, it is reasonable to group all the non-lesion liver tissue together for comparison. Another point worth mentioning is the diameters of most lesions in our study were between $1 \mathrm{~cm}$ and 2 $\mathrm{cm}$, a size with low diagnosis accuracy in dynamic CT and static PET [4, 37], which demonstrated the advantage of our method in differentiation of small liver lesions.

\section{CONCLUSIONS}

The ability of liver lesion differentiation and staging with parameters from static PET imaging analysis is limited. ${ }^{11} \mathrm{C}$-acetate kinetic modeling with a dual input provides a better view of the tracer behavior and more quantitative parameters to characterize the metabolism, perfusion and blood supply of liver lesion. The discriminant analysis with $K_{1}$ and $\mathrm{T} / \mathrm{L}$ improves the accuracy of the differentiation and staging of HCC and benign lesions, which potentiates clinical diagnosis and therapy of liver masses. For the future clinical application of our approach, a further study with larger patient group may be necessary. Also the computation efficiency needs to be addressed for the calculation of kinetic parameters in daily clinical practice.

\section{SUPPLEMENTARY MATERIAL}

Fig.S1 - Fig.S3, Table S1.

http://www.thno.org/v05p0371s1.pdf

\section{ACKNOWLEDGMENTS}

This work was supported in part, by the National Natural Science Foundation of China (Grant No. $81071188,81371596)$, by National Key Basic Research Program (973 Project) (2013CB733802), and by the Intramural Research Program of the National Institute of Biomedical Imaging and Bioengineering (NIBIB), National Institutes of Health (NIH).

\section{COMPETING INTERESTS}

The authors have declared that no competing interest exists.

\section{REFERENCES}

1. Choi BY, Nguyen MH. The diagnosis and management of benign hepatic tumors. J Clin Gastroenterol. 2005; 39: 401-12.

2. Terkivatan T, Hussain SM, De Man RA, Ijzermans JNM. Diagnosis and treatment of benign focal liver lesions. Scand J Gastroentero. 2006; 41: 102-15.

3. Rampone B, Schiavone B, Martino A, Viviano C, Confuorto G. Current management strategy of hepatocellular carcinoma. World J Gastroenterol 2009; 15: 3210-6.

4. Ho CL, Yu SC, Yeung DW. 11C-acetate PET imaging in hepatocellular carcinoma and other liver masses. J Nucl Med. 2003; 44: 213-21.

5. Delbeke D, Pinson CW. 11C-acetate: a new tracer for the evaluation of hepatocellular carcinoma. J Nucl Med. 2003; 44: 222-3.

6. Park JW, Kim JH, Kim SK, Kang KW, Park KW, Choi JI, et al. A prospective evaluation of 18F-FDG and 11C-acetate PET/CT for detection of primary and metastatic hepatocellular carcinoma. J Nucl Med. 2008; 49: 1912-21. 
7. Sacks A, Peller PJ, Surasi DS, Chatburn L, Mercier G, Subramaniam RM. Value of PET/CT in the management of primary hepatobiliary tumors, part 2. AJR Am J Roentgenol. 2011; 197: W260-5.

8. Karhunen PJ. Benign hepatic tumours and tumour like conditions in men. J Clin Pathol. 1986; 39: 183-8.

9. Delbeke D, Martin WH, Sandler MP, Chapman WC, Wright JK, Jr., Pinson CW. Evaluation of benign vs malignant hepatic lesions with positron emission tomography. Arch Surg. 1998; 133: 510-5; discussion 5-6.

10. Gibbs JF, Litwin AM, Kahlenberg MS. Contemporary management of benign liver tumors. Surg Clin North Am. 2004; 84: 463-80.

11. Chen S, Ho C, Feng D, Chi Z. Tracer kinetic modeling of 11C-acetate applied in the liver with positron emission tomography. IEEE Trans Med Imaging. 2004; 23: 426-32.

12. Paone G, De Angelis G, Munno R, Pallotta G, Bigioni D, Saltini C, et al. Discriminant analysis on small cell lung cancer and non-small cell lung cancer by means of NSE and CYFRA-21.1. Eur Respir J. 1995; 8: 1136-40.

13. Huang D, Quan $Y$, He M, Zhou B. Comparison of linear discriminant analysis methods for the classification of cancer based on gene expression data. J Exp Clin Cancer Res. 2009; 28: 149.

14. Su J, Yoon BJ, Dougherty ER. Accurate and reliable cancer classification based on probabilistic inference of pathway activity. PloS one. 2009; 4: e8161.

15. Edmondson HA, Steiner PE. Primary carcinoma of the liver - a study of 100 cases among 48,900 necropsies. Cancer. 1954; 7: 462-503.

16. Zhou L, Rui JA, Ye DX, Wang SB, Chen SG, Qu Q. Edmondson-steiner grading increases the predictive efficiency of TNM staging for long-term survival of patients with hepatocellular carcinoma after curative resection. World J Surg. 2008; 32: 1748-56.

17. Mitterhauser M, Wadsak W, Krcal A, Schmaljohann I, Bartosch E, Eidherr H, et al. New aspects on the preparation of [11C]acetate--a simple and fast approach via distillation. Applied radiation and isotopes : including data, instrumentation and methods for use in agriculture, industry and medicine. 2004; 61: 1147-50.

18. Chen S, Feng D. Evaluation of hepatocellular carcinoma with dynamic 11C-acetate PET: A dual-modeling method. IEEE Trans Nucl Sci. 2008; 55: 999-1007.

19. Soret M, Bacharach SL, Buvat I. Partial-volume effect in PET tumor imaging. J Nucl Med. 2007; 48: 932-45.

20. [Internet] http://www.activexray.com/pdf/Siemens_Biograph.pdf.

21. Soloviev D, Fini A, Chierichetti F, Al-Nahhas A, Rubello D. PET imaging with $11 \mathrm{C}$-acetate in prostate cancer: a biochemical, radiochemical and clinical perspective. Eur J Nucl Med Mol Imaging. 2008; 35: 942-9.

22. Yoshimoto M, Waki A, Yonekura $\mathrm{Y}$, Sadato N, Murata T, Omata N, et al. Characterization of acetate metabolism in tumor cells in relation to cell proliferation: acetate metabolism in tumor cells. Nucl Med Biol. 2001; 28: 117-22.

23. Randle PJ, England PJ, Denton RM. Control of the tricarboxylate cycle and its interactions with glycolysis during acetate utilization in rat heart. Biochem J. 1970; 117: 677-95.

24. Swinnen JV, Van Veldhoven PP, Timmermans L, De Schrijver E, Brusselmans $\mathrm{K}$, Vanderhoydonc F, et al. Fatty acid synthase drives the synthesis of phospholipids partitioning into detergent-resistant membrane microdomains. Biochem Biophys Res Commun. 2003; 302: 898-903.

25. Zheng X, Wen L, Yu SJ, Huang SC, Feng DD. A study of non-invasive Patlak quantification for whole-body dynamic FDG-PET studies of mice. Biomed Signal Process Control. 2012; 7: 438-46.

26. Maroco J, Silva D, Rodrigues A, Guerreiro M, Santana I, de Mendonca A. Data mining methods in the prediction of Dementia: A real-data comparison of the accuracy, sensitivity and specificity of linear discriminant analysis, logistic regression, neural networks, support vector machines, classification trees and random forests. BMC Res Notes. 2011; 4: 299

27. Burn DJ, Sawle GV, Brooks DJ. Differential diagnosis of Parkinson's disease, multiple system atrophy, and Steele-Richardson-Olszewski syndrome: discriminant analysis of striatal 18F-DOPA PET data. J Neurol Neurosurg Psychiatry. 1994; 57: 278-84.

28. Wardak M, Schiepers C, Dahlbom M, Cloughesy T, Chen W, Satyamurthy N, et al. Discriminant analysis of $18 \mathrm{~F}$-fluorothymidine kinetic parameters to predict survival in patients with recurrent high-grade glioma. Clin Cancer Res. 2011; 17: 6553-62.

29. Winterdahl M, Keiding S, Sorensen M, Mortensen FV, Alstrup AK, Munk OL. Tracer input for kinetic modelling of liver physiology determined without sampling portal venous blood in pigs. Eur J Nucl Med Mol Imaging. 2011; 38: 263-70.

30. Jakab F, Rath Z, Schmal F, Nagy P, Faller J. Changes in hepatic hemodynamics due to primary liver tumours. HPB Surg. 1996; 9: 245-8.

31. Jakab F, Rath Z, Schmal F, Nagy P, Faller J. Blood flow measurement in patients with hepatocellular carcinomas. Acta Chir Hung. 1994; 34: 87-94.

32. Moriyasu F. Doppler ultrasound in diagnosis of liver tumor and portal hypertension. Radiol Med. 1993; 85: 44-55.

33. Asayama Y, Yoshimitsu K, Nishihara Y, Irie H, Aishima S, Taketomi A, et al. Arterial blood supply of hepatocellular carcinoma and histologic grading: radiologic-pathologic correlation. AJR Am J Roentgenol. 2008; 190: W28-34.

34. Liu YI, Shin LK, Jeffrey RB, Kamaya A. Quantitatively defining washout in hepatocellular carcinoma. AJR Am J Roentgenol. 2013; 200: 84-9.

35. Tomasi G, Turkheimer F, Aboagye E. Importance of quantification for the analysis of PET data in oncology: review of current methods and trends for the future. Mol Imaging Biol. 2012; 14: 131-46.
36. Coakley FV, Schwartz LH. Imaging of hepatocellular carcinoma: a practical approach. Semin Oncol. 2001; 28: 460-73.

37. Tateishi Y. Diagnosis of 26 small hepatocellular carcinoma using incremental dynamic computed tomography. Kurume Med J. 1997; 44: 321-6. 